\title{
A study of cyst rupture rate by tissue type in open vs. laparoscopic surgery for ovarian tumor
}

\author{
Masahiko Umemoto • Mitsuru Shiota • \\ Takako Tobiume • Yasushi Kotani • Hiroshi Hoshiai
}

Received: 25 March 2011 /Accepted: 8 June 2011 / Published online: 22 June 2011

(C) Springer-Verlag 2011

\begin{abstract}
Laparoscopy may be suitable for benign ovarian tumors, but the risk of intraoperative cyst rupture may carry serious consequences should the lesion prove cancerous postoperatively. Incidence of cyst rupture may be related to lesion tissue type; however, to our knowledge, no study to date has evaluated it by tissue type. We report herein a comparative retrospective study of open surgery and laparoscopy on cyst rupture by tissue type. A total of 1,483 cases of surgical removal of benign ovarian tumor performed at our institution between 1995 and 2010 were reviewed. Cyst rupture rates during open surgery and laparoscopy were compared for tumorectomy and adnexectomy for the four most frequent tissue types found at postoperative histopathology: endometrial cyst, mature cystic teratoma, serous cystadenoma, and mucinous cystadenoma. Data collected for our study demonstrated that cyst rupture rates are higher in laparoscopy than in open surgery regardless of tissue type. Rupture rates were significantly higher during laparoscopic tumorectomy, while there was no significant difference overall in rupture rate for open vs. laparoscopic adnexectomy. Laparoscopic procedures should be performed with utmost care to avoid cyst rapture when malignancy cannot be ruled out. A high risk of cyst rapture preoperatively expected by tissue type dictates an open surgery.
\end{abstract}

Keywords Ovarian tumor. Cyst rupture rate - Open surgery $\cdot$ Laparoscopic surgery

M. Umemoto $(\bowtie) \cdot$ M. Shiota $\cdot$ T. Tobiume $\cdot$ Y. Kotani $\cdot$

H. Hoshiai

Department of Obstetrics and Gynecology,

Kinki University School of Medicine,

377-2 Ohno-higashi, Osaka-sayama,

Osaka 589-8511, Japan

e-mail: m-umemoto@sanfu.med.kindai.ac.jp

\section{Background}

Laparoscopy has become the treatment of choice for ovarian cyst in most facilities, because of its favorable impact on patients' QOL [1]. Nevertheless, there are concerns regarding the risk of intraoperative cyst rupture, should the lesion ultimately prove cancerous by postoperative histopathology. Some cases preoperatively diagnosed as benign may prove malignant postoperatively. Nezhat et al. have reported finding postoperative malignancy in $0.4 \%$ of cases [1]. Therefore, more strict preoperative differentiation is essential in guiding the choice of procedure.

Intraoperative cyst rupture is generally considered more frequent with laparoscopy than with open surgery. However, to our knowledge, no study has been conducted to evaluate cyst rupture rate by tissue type. We therefore completed a retrospective review of laparoscopic and open surgeries performed in our institution to examine cyst rupture rate by the tissue type and operation (tumorectomy vs. adnexectomy).

\section{Methods}

A total of 1,483 benign ovarian tumors were surgically removed at our center between 1995 and 2010, among which endometrial cyst, mature cystic teratoma, serous cystadenoma, and mucinous cystadenoma were the four most frequent types confirmed on postoperative histopathology. Intraoperative cyst rupture rates in tumorectomy and in adnexectomy for these tumor types were compared between laparoscopy and open surgery. A "no-rupture" case in laparoscopy was defined as one in which the tumor or ovary was extracted intact and removed from the body using a tissue collection bag, without leakage of content into the abdominal cavity. Extracorporeal and alcohol fixation 
Table 1 Cyst rupture rates during laparoscopic surgery and open surgery were compared for tumorectomy

\begin{tabular}{lllr}
\hline & Laparoscopic surgery & Open surgery & $P$ value \\
\hline Endometrial cyst & $94.8 \%(363 / 383)$ & $88.4 \%(84 / 95)$ & 0.02 \\
Mature cystic teratoma & $76.6 \%(219 / 286)$ & $28.6 \%(26 / 91)$ & $<0.01$ \\
Serous cystadenoma & $64.1 \%(25 / 39)$ & $33.3 \%(5 / 15)$ & 0.04 \\
Mucinous cystadenoma & $75.0 \%(24 / 32)$ & $50.0 \%(4 / 8)$ & 0.17 \\
Total & $82.8 \%(682 / 824)$ & $56.8 \%(138 / 243)$ & $<0.01$ \\
\hline
\end{tabular}

cases were excluded, as were cases in which the tumor volume was decreased by aspiration. The cases converted from laparoscopy to open surgery were also excluded. In other words, only cyst ruptures resulting inevitably from laparoscopy performed as planned were counted.

As usual at our institution, the laparoscopic procedure was performed with a $12-\mathrm{mm}$ trocar and three $5-\mathrm{mm}$ trocars inserted respectively at the umbilicus, in the lower abdominal midline, and to the right and left sides of the midline following closed pneumoperitoneum through the umbilicus. Adnexectomy was performed by removing the adnexa after ligating the fallopian tubes and the ovarian proper ligament and doubly ligating the ovary suspensory ligament in both open surgery and laparoscopy. In ovarian tumorectomy, the ovary surface was incised halfway around the equatorial plane from the opposite side of the hilum to extract the tumor while avoiding cyst rupture. A collection pouch (Endocatch gold ${ }^{\circledR}$ ) was inserted with a 12-mm trocar to collect the excised tumor. The tumor content was aspirated within the pouch, which was then carried out of the body.

For statistical analysis, the results were compared using the chi-square test, and $P<0.05$ was considered as significant.

\section{Findings}

Of the 1,483 procedures, 986 were laparoscopic, and 497 were open. The four most frequent tumor types among all procedures were endometrial cyst (551), mature cystic teratoma (510), serous adenoma (121), and mucinous adenoma (111).

Cyst rupture rates in tumorectomy for endometrial cyst were $94.8 \%$ and $88.4 \%$, respectively, for laparoscopy and open surgery; in adnexectomy, cyst rupture rates were
$80.0 \%$ and $47.2 \%$, respectively. Statistical analysis showed significantly higher rates of cyst rupture in laparoscopy for both (Tables 1 and 2). The rupture rates in tumorectomy for mature cystic teratoma were $76.7 \%$ and $28.6 \%$, respectively, for laparoscopy and open surgery, and those in adnexectomy were $16.1 \%$ and $4.2 \%$. Statistical analysis showed significantly higher rates in laparoscopy for both approaches. Cyst rupture rates in tumorectomy for serous adenoma were $64.1 \%$ and $33.3 \%$, respectively, which proved a significantly higher rupture rate for laparoscopy, while those in adnexectomy were $11.1 \%$ and $10.0 \%$, where no significant difference was found. No significant difference was found in cyst rupture rates in tumorectomy for mucinous adenoma, which were $74.0 \%$ and $50.0 \%$, respectively, for laparoscopy and open surgery, while those in adnexectomy were significantly different at $28.6 \%$ and $7.0 \%$, respectively. Overall results for all tissue types demonstrated a significantly higher rupture rate for laparoscopy particularly during tumorectomy ( $82.8 \%$ vs. $56.8 \%$ open), while during adenexectomy, there was no significant difference overall in tumor rupture rates, which were $22.2 \%$ and $15.7 \%$, respectively. This study selected only the cases preoperatively assumed benign. However, postoperative pathology revealed malignancy in $0.9 \%(13 / 1,483)$ of the cases and borderline malignancy in $1.5 \%$.

\section{Discussion}

Laparoscopy performed for a preoperatively diagnosed benign cyst, later confirmed as early ovarian cancer, may cause several problems including upstaging due to intraoperative cyst rupture, difficulty in precise staging due to insufficient sample collection, possible delayed initial treatment, metastasis/recurrence at the trocar insertion site,
Table 2 Cyst rupture rates during laparoscopic surgery and open surgery were compared for adnexectomy

\begin{tabular}{lccc}
\hline & Laparoscopic surgery & Open surgery & $P$ value \\
\hline Endometrial cyst & $80.0 \%(16 / 20)$ & $47.2 \%(25 / 53)$ & 0.01 \\
Mature cystic teratoma & $16.1 \%(10 / 62)$ & $4.2 \%(3 / 71)$ & 0.02 \\
Serous cystadenoma & $11.1 \%(3 / 27)$ & $10.0 \%(4 / 40)$ & 0.88 \\
Mucinous cystadenoma & $28.6 \%(4 / 14)$ & $7.0 \%(4 / 57)$ & 0.02 \\
Total & $22.2 \%(36 / 162)$ & $15.7 \%(40 / 254)$ & 0.10 \\
\hline
\end{tabular}


and tumor development/extension caused by $\mathrm{CO}_{2}$ pneumoperitoneum. The probability of prognostic upstaging as a result of intraoperative tumor rupture remains controversial $[2,3]$. Ahmed et al. have reported that intraoperative cyst rupture has no prognostic impact [4], while Canis et al. have regarded intraoperative tumor rupture as an important prognostic factor in stage I ovarian cancer and have recommended that it should be avoided as frequently as possible, even for locally limited ovarian cancers in which the risk of laparoscopic cyst rupture is very low [5]. Vergote et al. have also urged that intraoperative cyst rupture should be avoided in the initial surgery for locally limited ovarian cancer because their findings have indicated that the preoperative or intraoperative cyst rupture affects prognosis [2]. Other recent reports have stated that intraoperative cyst rupture has negative effects on 5-year disease-free survival and other outcomes. On the basis of these observations, it becomes clear that even with preoperative evidence supporting benign cyst, intraoperative cyst rupture should be avoided in all cases when possible.

\section{Conclusion}

Our retrospective study has demonstrated that cyst rupture rate was higher in laparoscopy than in open surgery in most of the tissue types observed. Despite the most meticulous caution, cyst rupture during either approach remains unavoidable at times. The goal of laparoscopic surgery is to resect the tumor or ovary with no rupture, collecting the resected mass in a pouch and aspirating the tumor content there, and carrying it out of the body. Success in this process may be hindered by factors including abdominal adhesions and the size of the surgical collection bag, which may contribute to a higher incidence of cyst rupture in laparoscopy than in open surgery. Therefore, it is necessary to choose an operative procedure (i.e., laparoscopic vs. open; tumorectomy vs. adenexectomy) based on assessments drawn from preoperative diagnostic imaging. When the preoperative diagnostic images show solid portion or thickened septum, the tumor has high probability of malignancy regardless of its size [6]. Laparoscopic procedures should be performed with utmost care to avoid cyst rapture when possibility of malignancy cannot be denied. When a high risk of cyst rapture is expected preoperatively by tissue type, open surgery should be selected.

Declaration of interest The authors report no conflicts of interest. The authors alone are responsible for the content and writing of the paper.

\section{References}

1. Nezhat F, Nezhat C, Welander CE, Benigno B (1992) Four ovarian cancers diagnosed during laparoscopic management of 1011 women with adnexal masses. Am J Obstet Gynecol 167:790-796

2. Vergote I, De Brabanter J, Fyles A et al (2001) Prognostic importance of degree of differentiation and cyst rupture in stage I invasive epithelial ovarian carcinoma. Lancet 357:176-182

3. Dembo AJ, Davy M, Stenwig AE, Berle EJ, Bush RS, Kjorstad K (1990) Prognostic factors in patients with stage I epithelial ovarian cancer. Obstet Gynecol 75:263-273

4. Ahmed FY, Wiltshaw E, A'Hern RP et al (1996) Natural history and prognosis of untreated stage I epithelial ovarian carcinoma. J Clin Oncol 14:2968-2975

5. Canis M, Rabischong B, Houlle C et al (2002) Laparoscopic management of adnexal masses: a gold standard? Curr Opin Obstet Gynecol 14:423-428

6. Umemoto M, Shiota M, Shimono T, Hoshiai H (2006) Preoperative diagnosis of ovarian tumors, focusing on the solid area based on diagnostic imaging. J Obstet Gynaecol Res 32:195-201 